\title{
Hybrid Strategy for Routing, Modulation and Spectrum Assignment in Elastic Optical Networks
}

\author{
Karcius D. R. Assis - Alex F. dos Santos - Raul \\ C. Almeida Jr • Thiale M. A. de Oliveira • Rafael \\ A. Vieira - Leonardo A. J. Mesquita - Felipe P. \\ Correia - Helder A. Pereira
}

Received: date / Accepted: date

\begin{abstract}
In this paper, we propose a novel hybrid strategy that combines mixed integer linear programming (MILP) formulation with different alternative routing approaches that is capable of solving, simultaneously, the routing, modulation format and spectrum assignment problem in the design of elastic optical networks. We extend our proposal to a path-link formulation that, although it is not guaranteed to find the optimal solution, it has shown reasonable solutions within inferior simulation time, allowing it to be used in not very short networks. We also compare our proposal with another MILP formulation and three adapted heuristics, all available in the literature. The results show the benefits of our proposal considering diverse simulation scenarios and different number of modulation formats over 19 realistic networks in terms of simulation run time and maximum number of used set of slots.
\end{abstract}

Keywords Mixed Integer Linear Programming · Optical Network · Routing, Modulation Level and Spectrum Assignment · Static Traffic

\section{Introduction}

The routing and spectrum assignment (RSA) problem (Wang et al., 2011), plays an important role in design of modern elastic optical networks (EON) (Chatterjee and Oki, 2020; Alyatama et al.,

Karcius D. R. Assis - Thiale M. A. de Oliveira - Rafael A. Vieira · Leonardo A. J. Mesquita

Federal University of Bahia (UFBA), Department of Electrical Engineering, Salvador, Bahia, Brazil.

E-mail: karcius.assis@ufba.br

Alex F. dos Santos

Science and Technology Center on Energy and Sustainability, University of Recôncavo of Bahia (UFRB), Feira de Santana, Bahia, Brazil.

E-mail: alex.ferreira@ufrb.edu.br

Raul C. Almeida Jr

Photonics Group, Electronics and Systems Department, Technology Center and Geosciences, Federal University of Pernambuco (UFPE), Recife, Pernambuco, Brazil.

E-mail: raul.almeidajunior@ufpe.br

Felipe P. Correia

Federal Institute of Sertão Pernambucano (IFSERTAO-PE), Petrolina, Pernambuco, Brazil.

E-mail: felipe.correia@ifsertao-pe.edu.br

Helder A. Pereira

Electrical Engineering Department, Electrical Engineering and Informatics Center, Federal University of Campina Grande (UFCG), Campina Grande, Paraiba, Brazil.

E-mail: helder.pereira@dee.ufcg.edu.br 
2020; Xuan et al., 2017). It is very similar to the routing and wavelength assignment (RWA) problem well-described in the literature of wavelength division multiplexing (WDM) networks Ramaswami et al. (2009). The main difference basically corresponds to the flexible bandwidth assigned to the call requests in comparison to the fixed bandwidth considered in WDM networks. RSA and RWA problems are concerned with the search for a route with available network resources to attend each network call request. The practical planning of both strategies requires a solution with resource saving (Wang et al., 2011; Klinkowski and Walkowiak, 2011; Christodoulopoulos et al., 2010; Zang et al., 2000).

Many papers in the literature have attempted to assess the significance of saving frequency spectrum in the prevention of network capacity crunch (Chralyvy, 2009; Waldman, 2018; Mavridopoulos et al., 2020). The granularity and correct elastic bandwidth assignment are challenges to be overcome when designing EON optimization algorithms. The network planner must have the possibility to evaluate the frequency spectrum usage and the costs of different network configurations, considering peculiar characteristics and constraints (Cavalcante et al., 2017). To reduce the cost of EONs' design and to guarantee the traffic attendance, the network planner can exploit the heterogeneity in the transmission systems so that some long-distance high transmission bit-rate paths are assigned to a modulation format having high bandwidth-distance product (Zhao et al., 2015). Unfortunately, higher spectral efficiency modulation format would only allow shorter transmission distances due to network physical impairments (Zhao et al., 2015). Therefore, when more than one modulation format is available in the network, the complexity of optimization techniques increases drastically (Christodoulopoulos et al., 2011; Velasco et al., 2012). This problem is called routing, modulation level and spectrum assignment (RMLSA) in the literature (Varvarigos, 2013). Some strategies have already been proposed to solve the RMLSA problem to achieve optimal results, but the formulation complexity has shown to be even higher than the RSA problem (without modulation format constraints), making processing time intractable for realistic network applications (Klinkowski et al., 2011). Methods based on heuristics (Yin et al., 2013a; Savory, 2014) and meta-heuristics, such as evolutionary-based algorithms (Gong et al., 2012) and deep neural networks (Chen et al., 2018), have been tested to find sub-optimal solutions considering integer linear programming (ILP) formulations applied to the RMLSA problem. However, researchers have not treated in much details the solution of the problem for several networks with different caractheristics (size, physical connectivity etc).

Therefore, the design of EONs incorporating modulation equipment required the development of models that take into account the limitations imposed by these tecnnologies and by the physical substrate as well. Besides, the particular nature of the transported traffic. The key issue in the EONs design process once the physical realization has been achieved, is to properly dimension the network with respect to slots number and to determine a frequency allocation plan.

In this paper, we address jointly optimization of modulation format and spectrum assignment. The network planning problems can be solved offline since the network is not yet in operation and hence generally no strict time limit is applied to solve those problems. In contrast, when the network is in operation, stringent solving times are usually necessary. Focusing on both, offline and in-operation planning, the contribution of this paper is a hybrid MILP formulation that can be used to model network planning variant of the RMLSA problem, named PSP-MILP formulation (MILP with predefined set of paths), with optimized paths using any alternative routing algorithm (in this paper, Yen algorithm (Katoh et al., 1982)).

The overall structure of the study takes the form of six sections, including this introductory section. Section 2 is a brief explanation and literaure review about the RMLSA problem. Section 3 explains about the tradittional formulation approaches. Section 4 begins by laying out the theoretical dimensions of the research and is concerned with hybrid formulation. Section 5 presents the findings of the research, focusing on a small and several large networks. Finally, Section 6 gives a brief summary, a critique of the findings and identifies areas for further research. 


\section{Routing, Modulation Level and Spectrum Assignment}

In this section, we describe in details the RMLSA problem, the literature review of EONs under static traffic, a complete MILP formulation, extracted from (Oner, 2016), and three adapted heuristics from the literature (Wang et al., 2011; Durães et al., 2010) to be jointly used with our proposed hybrid MILP strategy.

\subsection{The RMLSA Problem}

In transparent EONs, data is routed along the links without going through any optical-electricaloptical conversion (Gerstel et al., 2012). If it is not possible to do frequency spectrum conversion when a call request is routed between a source and a destination node, the same frequency slots (FS) must be used throughout the set of links that composes the lightpath. This condition is known in the literature as continuity constraint (Christodoulopoulos et al., 2011; Oner, 2016). Another point related to EONs is that, for a single call request, the assigned FSs must be adjacent to one another, for efficiency sake. This is the contiguity constraint (Christodoulopoulos et al., 2011; Oner, 2016). This paper adopts both continuity and contiguity constraints.

The quality of transmission (QoT) of a specific call request depends on the distance and the transmission bit rate. Thus, the chosen modulation format must be such that the signal QoT after the signal propagates along the route links is above the minimum QoT required by the modulation format (modulation format constraint). Therefore, whenever a call request must be assigned to a route between two nodes in an EON, these three constraints must be considered, which together constitute the main functions of the RMLSA problem (Christodoulopoulos et al., 2011). Thus, RMLSA problem becomes more intricate because the routing must take into account which is the best path to take, relative to the available modulation formats and their respective physical distances (Christodoulopoulos et al., 2010).

Consider that each FS occupies $\Omega \mathrm{GHz}$ of bandwidth and that a demand of $v$ Gbps is admitted with a modulation format with spectral efficiency $\eta$ Gbps per GHz. The number of FSs necessary to attend such call request is given by (1).

$$
p=\left\lceil\frac{v}{\Omega \eta}\right\rceil,
$$

in which $\lceil x\rceil$ is the upper integer closest to $x$ (ceil operator). If the spectral efficiency $(\eta)$ of the modulation format is increased, the number of required slots $(p)$ is decreased. Nevertheless, a higher spectral efficiency implies a shorter transmission distance.

Fig. 1 shows an example of a RMLSA solution considering a four-node ring network. The nodes and the links are plotted in black. The nodes are numbered from 1 to 4 . The paths from the source to the destination node, through which the traffic flows, are represented by directional arrows. The set of FSs for each of the eight links $\left(e_{i j}\right)$ are on the left and right sides of Fig. 1. The blue filled boxes are the FSs that were assigned to a traffic demand $\left(v^{i j}\right)$ indicated above the box. The grey colored boxes without indication are considered as guard bands.

Suppose that the traffic matrix $V=\left\{v^{i j} \in N_{4 \times 4}\right\}$, in which the elements $v^{i j}$ represent the traffic demand from node $i$ to node $j$ in Gbps, whose values are described by (2), must be attended by the network shown in Fig. 1.

$$
V=\left[\begin{array}{cccc}
0 & 50 & 50 & 25 \\
70 & 0 & 65 & 30 \\
60 & 120 & 0 & 45 \\
100 & 35 & 150 & 0
\end{array}\right]
$$




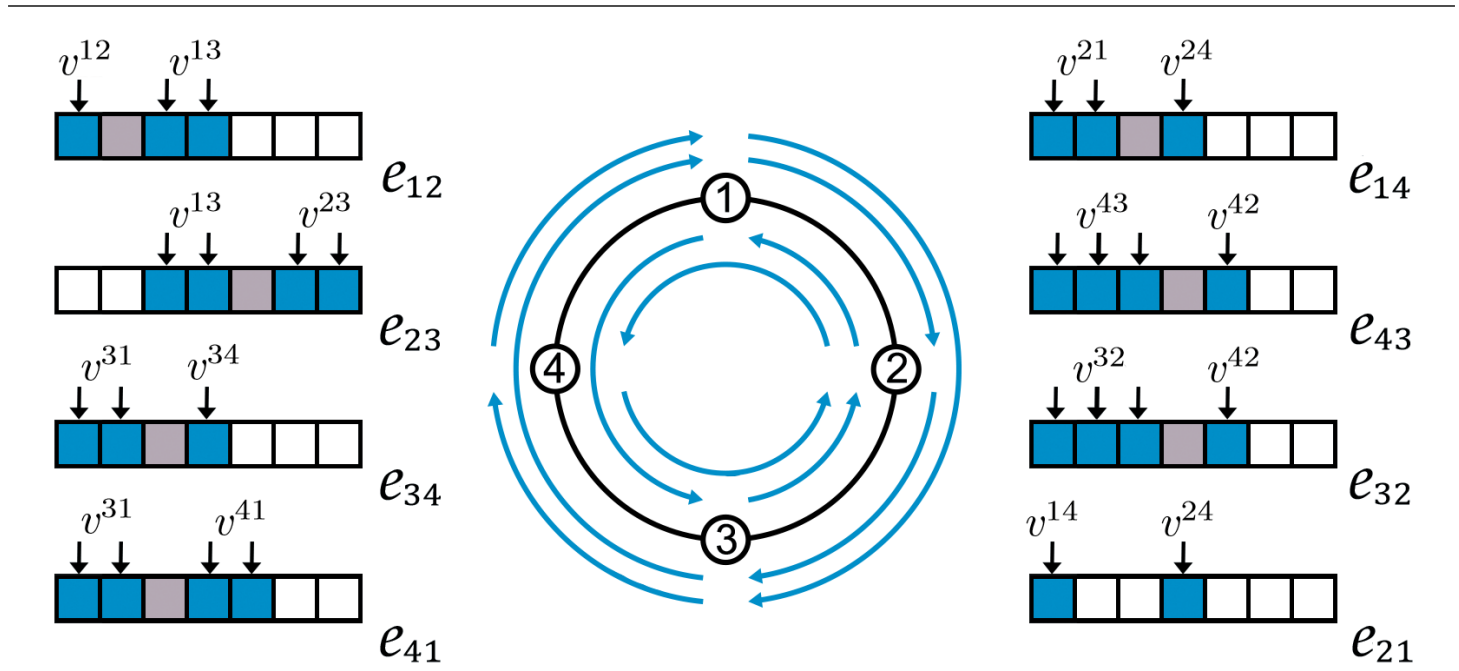

Fig. 1: Example of a RMLSA solution considering a four-node ring network.

For the example illustrated in Fig. 1, it was considered that: $(a)$ the strategy that solves the RMLSA problem for the four-node ring network is a heuristic that always chooses the shortest path to route the emerging demand; $(b)$ just the modulation formats 8-QAM and 16-QAM can be used; $(c)$ every link has a distance of $500 \mathrm{~km} ;(d)$ the policy that dictates at which position to assign the set of FSs always chooses the first free slots $(\mathrm{FF})$; and $(e)$ exactly one guard band should be reserved.

The heuristic picks the traffic elements in a particular order, from the traffic matrix. In this example, elements will be chosen first from left to right, and then from top to bottom (reading pattern). The first valid element to assign is the $50 \mathrm{Gbps}$ demand from node 1 to node 2 . The shortest path uses link $e_{12}$. Since the total distance is equal to $500 \mathrm{~km}$, it is possible to use 16 QAM as modulation format. Thus, applying (1), a single slot is assigned for the link $e_{12}$. The next demand is from node 1 to node 3 with $50 \mathrm{Gbps}$. There are two shortest paths, so the heuristic can choose any of these two paths. The sequence of nodes that participates in the path is $1 \rightarrow 2 \rightarrow 3$. The total distance is equal to $1000 \mathrm{~km}$. Thus, the best modulation format that can be used is 8QAM and two contiguous FSs must be assigned continuously throughout links $e_{12}$ and $e_{23}$. Note, in Fig. 1 , that the demand $t_{13}$ had to be assigned after the guard band (slot 2 ) in link $e_{12}$. For this reason, in link $e_{23}$, the first two slots were left unassigned. This is in accordance with the continuity constraint, which forces the algorithm to use the same set of slots in all links, for the same call request. Following the same pattern to the other static traffic matrix elements, at the end, the RMLSA solution produced by the heuristic will be as presented in Fig. 1. Notice that, in link $e_{23}$, only the first slot can be occupied, because the second slot must be used as guard band. In addition, in link $e_{21}$, the second and third slots cannot be assigned at all, since there is no way to insert a demand (even with a single slot) alongside the two required guard band slots. This phenomenon, in which free scattered FSs become virtually useless, is called spectrum fragmentation (Yin et al., 2013b; Wang, 2019; Hua and Qiu, 2020; Adhikari et al., 2020).

\subsection{Literature Review}

In practice, before deploying a EON, certain activities need to be undertaken and there are a lot of works about this. Previous studies, as presented in (López et al., 2016), have reported demonstration of EONs planning and it is a clear illustration of the importance of the topic. The authors present several studies with MILP and heuristics for the design of EONs. Most studies starting with inputs 
from the service layer and from the state of the already deployed resources. A designing phase needs to be carried out to produce recommendations that can be used to design the network for a given period of time. After that, the network design is verified and manually implemented. While the network is in operation, its capacity can be continuously monitored and the resulting data is used as input for the next planning cycle (López et al., 2016; Mesquita et al., 2020). In case of unexpected increase in demand or network changes, nonetheless, the planning process may be restarted. To do so, traffic demands need to be routed over the network. Note that the RMLSA problem is a part of the optimization problem that needs to be solved over several aspects and there is a broad literature about that. Recently, (Yaghubi-Namaad et al., 2018; Lira et al., 2020).

Some studies of EONs explore the RMLSA problem using ILP formulation. In some papers (Klinkowski et al., 2011; Abkenar and Rahbar, 2017; Zhao et al., 2015; Gong et al., 2013) new ILP formulations and heuristics were proposed to minimize the bandwidth, i.e., the number of used set of slots across the network, while satisfying the demands on throughput and quality for all call requests.

Ríos et al. (2018) proposed an ILP model considering serial optimization (RML+SA) and linkoriented routing. The proposal, among all tested models, was one of those that converged to the best solution given its division into phases. Evolutionary algorithms such as genetic (Lechowicz and Walkowiak, 2016) and tabu search (Goścień et al., 2015) were proposed to solve the RMLSA offline problem and to enhance the spectrum utilization. Wang et al. (2015), in order to balance the optimality and complexity of the algorithm, proposed a recursive MILP formulation that yielded a suboptimal yet comparable solution to the MILP by using a lower and more consistent running time. Assis et al. (2019) proposed a novel and unified MILP formulation for dedicated path protection and two new protection schemes in EONs with multiple service-level agreements, grooming, and RSA. The proposed formulation enabled different survivability levels for the network traffic demands subject to committed service profiles, including bandwidth squeezing for each source and destination node pair, which could increase the number of surviving paths in the network at the price of reducing the traffic bandwidth under a link failure.

\section{MILP Formulation and Heuristics from Literature}

The RMLSA problem can be solved optimally to minimize a desired performance metric with the use of MILP formulations (Wang et al., 2015; Assis et al., 2019). Although MILP formulations can provide the best possible result, RMLSA is a NP-complete problem (Christodoulopoulos et al., 2011). Basically, it means that the execution time grows exponentially as a function of the number of nodes and links. Because of that, it is impractical to use these formulations for larger networks. Thus, in this paper, we have adapted the MILP formulation (Oner, 2016) to include pre-defined paths, from three different heuristics in the literature (SPSR, BLSA and BSR) (Wang et al., 2011; Durães et al., 2010), as well as added modulation format constraints with the aim of alleviating the run time for larger networks. This hybrid MILP approach is referred to as pre-defined shortest paths MILP formulation (PSP-MILP).

Prior to commencing the PSP-MILP study, the Subsection 3.1 provides a detailed description of the complete RMLSA MILP formulation, that uses balance equations instead of a pre-defined set of paths, therefore referred here to as non predefined set of paths (NPSP-MILP). We also present the adapted heuristics (Subsection 3.2 to Subsection 3.4). All these approaches will be compared to the proposed PSP-MILP formulation in order to show the superior performance of PSP-MILP when compared to the heuristics, and its reduced computational time when compared to NPSP-MILP formulation. 


\subsection{NPSP-MILP Formulation}

The complete MILP formulation presented in this subsection is an adaptation of (Assis et al., 2019) in which grooming and protection mechanisms equations were removed. Since NPSP-MILP uses flow balance equations, it is guaranteed to find the best routes to be used by any demand in the network. Therefore, NPSP-MILP can find the best possible RMLSA solutions that minimize the spectral use and save resources. However, it cannot provide its optimum solution in reasonable time for larger networks. The parameters, variables, objective function, modulation format, routing on physical topology, spectrum continuity and contiguity constraints, all related with NPSP-MILP, are presented as follows:

\section{Parameters}

- E: set of graph $G$ with physical links $(m-n)$ and $(n-m)$.

- $D$ : set of demands $i j$. From node $i$ to node $j$.

$-d_{m n}$ : length of a link $(m-n)$ on the physical topology.

$-v^{i j}$ : traffic intensity (in Gbps) for each demand $i j$.

- $M$ : number of available modulation formats.

$-\eta_{z}$ : spectral efficiency of modulation format $z$, in which $z \in 1, \ldots, M$.

$-d_{z}$ : lightpath maximum reach using modulation format $z$, in which $z \in 1, \ldots, M$.

$-\chi$ : a large number.

- $F$ : the frequency guard band in terms of number of slots.

\section{Variables}

- $p_{i j}$ : number of slots from node $i$ to node $j$.

$-P_{m n}^{i j}$ : route in terms of number of slots from node $i$ to node $j$ throughout a fiber link $(m-n)$.

$-A_{m n}^{i j}$ : binary variable that is equal to 1 , if $P_{m n}^{i j}>0$, and is equal to 0 if $P_{m n}^{i j}=0$.

$-\epsilon_{i j}^{z}$ : binary variable that is equal to 1 , if the modulation format $z$ is used, and 0 otherwise.

$-S_{i j}$ : binary variable that represents the starting frequency of connection $i j$.

- $W_{i j, k t}$ : binary variable that is equal to 1 , if $S_{i j}<S_{k t}$, and 0 otherwise.

$-C$ : maximum number of frequency slots.

\section{Objective function}

The objective function, described by (3), intends to minimize the highest slot index used in any link of the network.

\section{Minimize : $C$}

\section{Modulation format constraints}

The restrictions described by (4) and (5) determine the number of required slots for the lightpath given an assigned modulation format. Whenever $z$ is not the chosen modulation format, $\epsilon_{i, j}^{z}=0$ and the trivial solution $p_{i j}>-\infty$ is found. On the other hand, for the chosen modulation format, $\epsilon_{i, j}^{z}=1$ and the number of slots, which is an integer value, is calculated as $\frac{V_{i j}}{\Omega \eta_{z}} \leq p_{i j} \leq \frac{V_{i j}}{\Omega \eta_{z}}+1$.

$$
\begin{gathered}
p_{i j} \geq\left(\frac{v^{i j}}{\Omega \eta_{z}}\right)-\left(1-\epsilon_{i j}^{z}\right) \chi \quad \text { for } z=1,2, \ldots, M \\
p_{i j} \leq\left(\frac{v^{i j}}{\Omega \eta_{z}}\right)+1+\left(1-\epsilon_{i j}^{z}\right) \chi \quad \text { for } z=1,2, \ldots, M
\end{gathered}
$$

The distance limitation and the modulation format are adopted in a lightpath with restrictions (6) to (9). (6) asserts that $p_{i j}=0$ whenever no modulation format is assigned. (7) and (8) assure that at least one modulation format is assigned to the connection whenever traffic is assigned to it, i.e $v_{i j} \neq 0$. (9) assures that the distance limitation of the chosen modulation format is satisfied. 


$$
\begin{gathered}
p_{i j} \leq \chi \sum_{z} \epsilon_{i j}^{z} \\
\sum_{z} \epsilon_{i j}^{z} \leq 1 \\
\sum_{z} \epsilon_{i j}^{z} \geq \frac{v^{i j}}{\chi} \\
\sum_{m n} A_{m n}^{i j} d_{m n} \leq \sum_{z} d_{z} \epsilon_{i j}^{z}
\end{gathered}
$$

\section{Routing on physical topology}

Routing in the optical layer is treated by (10), which establishes a constraint on flow conservation.

$$
\sum_{n} P_{m n}^{i j}-\sum_{n} P_{n m}^{i j}=\left\{\begin{array}{rr}
p_{i j} & m=i \\
-p_{i j} & m=j \\
0 & m \neq i, j
\end{array} \quad \forall i j, m\right.
$$

The used bandwidth must not exceed the spectrum capacity of the fiber, including frequency band guard $(F)$. The restriction referring to the evaluation of the frequency band guard overload and the restriction that guarantees that the lightpath traffic cannot be partitioned in the physical topology are expressed by (11) and (12).

$$
\begin{aligned}
A_{m n}^{i j} & \geq \frac{P_{m n}^{i j}}{\chi} \quad \forall i j, m n \\
A_{m n}^{i j}+A_{m l}^{i j} & \leq 1 \quad \forall i, j, m ; \quad n \neq l
\end{aligned}
$$

The initial frequency of a call request and its slot numbers must no exceed the link capacity (in terms of slots), according to (13).

$$
p_{i j}+S_{i j} \leq C \quad \forall i j
$$

\section{Spectrum continuity and contiguity constraints}

In the event that lightpaths $i j$ and $k t$ share a fiber in the network, $A_{m n}^{i j}=A_{m n}^{k t}=1$ and either $W_{i j, k t}=1$ and $W_{k t, i j}=0$ or the opposity. Based on this, $W_{i j, k t}+W_{k t, i j}=1$. Accordingly, if $W_{i j, k t}=1$, $W_{k t, i j}=0$, and vice versa. Based on this and with (14) to (18), the frequency $W_{i j, k t}+W_{k t, i j}=1$ and the frequency spectrum cannot be overlapped. On the contrary, $W_{i j, k t}$ and $W_{k t, i j}$ can both be equal to zero if the lightpaths $i \rightarrow j$ and $k \rightarrow t$ do not share fibers. Thus, $A_{m n}^{i j}$ and $A_{m n}^{k t}$ cannot be equal to 1 simultaneously, consequently, there is no restriction on spectrum overlap.

$$
\begin{gathered}
S_{i j} \geq 0, p_{i j} \geq 0 \quad \forall i j \\
W_{i j, k t}+W_{k t, i j} \leq 1 \quad \forall i j, k t: i j \neq k t \\
W_{i j, k t}+W_{k t, i j} \geq\left[\left(A_{m n}^{i j}+A_{m n}^{k t}\right)-1\right] \quad \forall i j, k t: i j \neq k t \\
p_{i j}+S_{i j}+F \leq S_{k t}+\chi\left[1-W_{i j, k t}\right] \forall i j, k t: i j \neq k t \\
p_{k t}+S_{k t}+F \leq S_{i j}+\chi\left[1-W_{k t, i j}\right] \forall i j, k t: i j \neq k t
\end{gathered}
$$

The objective function (described by (3) and the restriction described by (13)) are used to minimize the maximum number of spectrum slots for all demands. 


\subsection{SPSR-M}

The shortest path with maximum spectrum reuse (SPSR) algorithm was proposed by Wang et al. (2011). SPSR originally aims to minimize the required slot number in EONs considering static traffic. It was proposed to solve the RSA problem in larger networks and combines the shortest path routing with the maximum reuse spectrum assignment (MRSA) algorithm. In MRSA, the spectrum of the lightpaths are first sorted according to the size of the traffic demand. Wang et al. (2011) considered that larger traffic demand has higher priority, only fiber-disjoint spectrum paths may reuse the same slots and employed a first-fit (FF) strategy to find available consecutive slots.

Considering a certain number of frequency slots (by using (1)) and a given demand $(d \subset D)$, it is possible to notice that the greater the reuse of the slot the greater the reduction in the maximum number of slots. Based on this idea, the SPSR was adapted in this paper to tackle with modulation format constraint (SPSR-M). Fig. 2 shows the flowchart of SPSR-M. Basically, each route is determined by Dijkstra algorithm and the most efficient modulation format available to this route is assigned. The set of routes is organized in a descending order in terms of number of slots and the set of slots are assigned to each route using FF algorithm.

\subsection{BLSA-M}

The balanced load spectrum assignment (BLSA) algorithm was also proposed by Wang et al. (2011). BLSA determines the routing by balancing the network load to potentially minimize the maximum slot number in the network links. First, BLSA uses the $k$-shortest path algorithm to generate $k$ routes for each network node-pair. Second, the route for each node-pair is decided with the goal of balancing the load among all network links. The candidate route that produces the smallest maximum link load is used as a route for the corresponding node-pair. BLSA considers the route with the highest traffic demand first, assigning one of the paths to it while minimizing the maximum link load, until all pairs of nodes with non-zero traffic demands are considered. Finally, it uses the MRSA algorithm to assign the slots in all routes. In this paper, BLSA algorithm was adapted to consider the modulation format constraint (BLSA-M). Fig. 2 shows the flowchart of BLSA-M.

\subsection{BSR-M}

The best among the shortest routes (BSR) algorithm was first proposed to plan WDM networks (Durães et al., 2010). The idea of BSR algorithm is to optimize the load balancing of the network links. It tries to find the best routing solution $\left(S_{i j}\right)$ among the set of fixed shortest paths $(D K)$. Through iterations, BSR algorithm adjusts the cost of each network link $C(l)$. This is done with a small weighting factor $(\alpha)$ proportional to the link utilization value $(u(l))$ obtained in previous iterations (it). The number maximum of iterations is ITm. BSR algorithm simulates a routing request and the results of each iteration are the values for each link and the performance of the spectrum slot number $\left(p_{i j}\right)$. In this paper, we have adapted the BSR first adapted to serve EONs (Assis et al., 2014) to tackle with modulation format constraint in static traffic considerations (BSR-M). Fig. 3 shows the flowchart of BSR-M.

\section{Our Proposal Hybrid MILP Formulation}

In the NPSP-MILP, presented by (3) to (18) (as described in Subsection 3.1), the modeling spans all feasible regions and fields in a global optimal solution for the RMLSA problem considering modulation format constraint. Although a global optimum is guaranteed, the solution time increases 


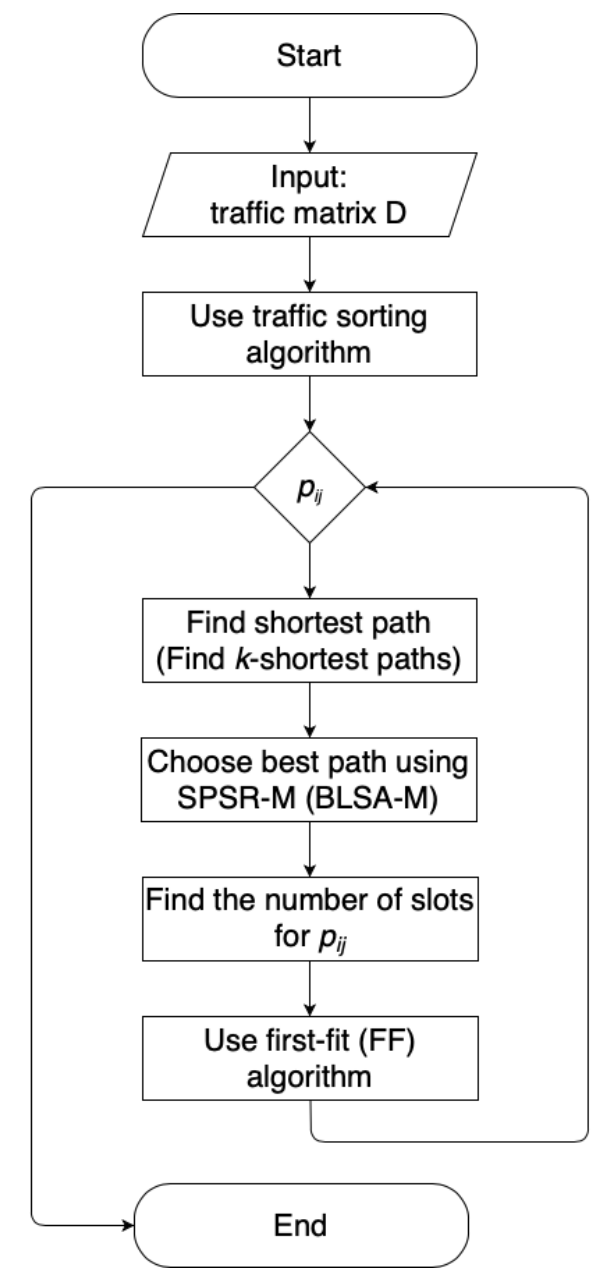

Fig. 2: Flowchart of SPSR-M (BLSA-M).

exponentially, which results impracticality for larger networks. In our proposal the constraints are created using link-path formulation, in which the paths are known beforehand. To find near optimal solutions, the PSP-MILP (therefore, with pre-defined paths) is used to substitute NPSPMILP in moderate (in terms of size) networks, considering different strategies of alternative routing algorithms (in this paper, Yen algorithm (Katoh et al., 1982)).

In comparison with NPSP-MILP, PSP-MILP requires some changes. Given the graph $G$ and the demand set $D$, the objective remains the same (minimize $C$ ), but PSP-MILP chooses a path of a source $i$ to a destination $j$ for each demand from the pre-determined set of routes $\left(P_{i j}\right)$ and a spectrum assignment that is the same in all links that the demand traverses in its chosen path. Additional parameters and variables are defined as follows:

\section{Parameters}

$-\beta_{i j, p}^{\alpha}=1$, if link $\alpha$ belongs to path $p$ realizing demand $i j$, and 0 otherwise.

$-P_{i j}$ : set of $k$ candidate paths for connection $i j$.

$-\mathcal{P}=\cup_{i j} P_{i j}$ : total set of candidate paths for demand set $D, i j \in D$.

\section{Variables}




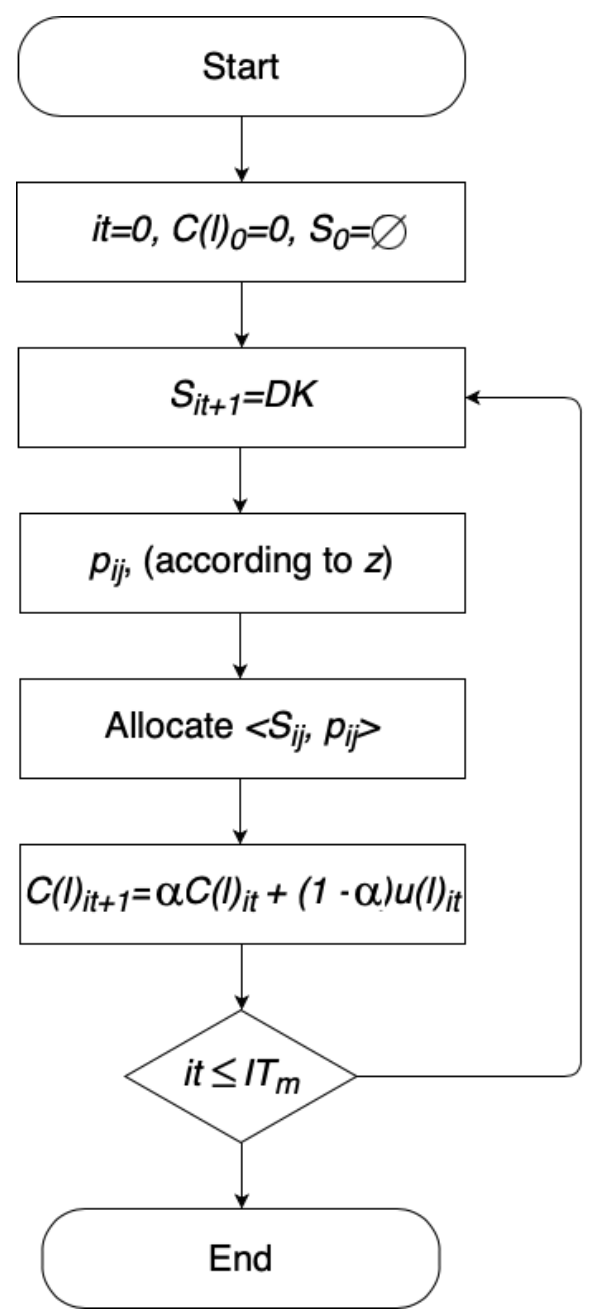

Fig. 3: Flowchart of BSR-M.

- $X_{i j, p}$ : binary variable that is equal to 1 , if path $p$ is used to demand $i j$, and 0 otherwise;

Therefore, if $\exists p \in P_{i j_{k}}$ and $\exists q \in P_{i j_{t}}$, such that $p \cap q \neq 0$, then for each $i j_{t}, i j_{k} \in D$ and every $p, q \in \mathcal{P}$. Then, the constraints described by (19) to (25) are employed instead of (3) to (8).

Paths topology constraints

$$
\begin{gathered}
\sum_{p \in P_{i j}} X_{i j, p}=1 \quad \forall i j \in D \\
\sum_{a} \beta_{i j, p}^{a} X_{i j, p} \leq \sum_{z} d_{z} \epsilon_{i j}^{z}
\end{gathered}
$$

Spectrum continuity and slot consecutiveness constraints

$$
W_{i j, k t}+W_{k t, i j}=1
$$




$$
\begin{gathered}
S_{i j}+p_{i j}+F-S_{k t} \leq \quad \chi\left[1-W_{i j, k t}+2-X_{i j, p}-X_{i j, q}\right] \\
S_{k t}+p_{k t}+F-S_{i j} \leq \quad \chi\left[1-W_{k t, i j}+2-X_{k t, p}-X_{k t, q}\right] \\
X_{i j, p} \in\{0,1\} \quad p \in \mathcal{P} \\
S_{i j} \geq 0 \quad i j \in D
\end{gathered}
$$

\section{Simulation Setup and Results Discussion}

In this section, we describe in details the simulation scenarios involving small (Subsection 5.1) and large (Subsection 5.2) networks, as well as the results obtained by our proposal (PSP-MILP) in comparison with NPSP-MILP and other heuristics adapted from the literature (SPSR-M, BLSA$\mathrm{M}$ and BSR-M).

For all simulations, the traffic matrices were generated as a matrix with the same dimensions as the network matrix. The simulation environment was set up on an Intel i7 computer with a 3.6 GHz processor and $32 \mathrm{~Gb}$ RAM. For the MILPs we use the solver CPLEX (Cplex, 2009).

\subsection{Small Network}

Fig. 4 shows the network with six nodes considered in our simulations as small network. The bandwidth slot was set at $12.5 \mathrm{GHz}(\Omega=12.5 \mathrm{GHz})$ and the guard band filter as well. The modulation formats available were varied from 1 to $3(1 \leq M \leq 3)$. Each modulation format has spectral efficiency equal to 1,2 and $3 \mathrm{bps} / \mathrm{Hz}$, respectively, in which $1 \leq \eta \leq 3$. The lightpath maximum reach under each modulation format $z$ is $d_{1}=4$ hops, $d_{2}=2$ hops and $d_{3}=1$ hop.

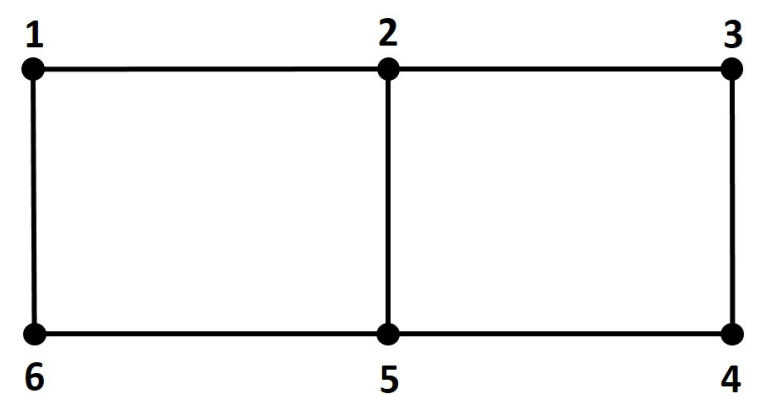

Fig. 4: Network with six nodes considered in our simulations as small network.

The run time analysis for a small network (shown in Fig. 4) was conducted only considering our proposed PSP-MILP jointly with Yen algorithm (with $k=1$ and $k=2$ ) in comparison to NPSP-MILP. The run time with heuristics (SPSR-M, BLSA-M and BSR-M) is around $1 \mathrm{~ms}$ for this first simulation scenario analyzed. The PSP-MILP, with $k=1$ and $k=2$, found a solution in a few seconds and NPSP-MILP in minutes or seconds, as described in details in Table 1. 
Table 1: Run time analysis for a small network (shown in Fig. 4) conducted only considering our proposed PSP-MILP jointly with Yen algorithm (Katoh et al., 1982) (with $k=1$ and $k=2$ ) in comparison to NPSP-MILP.

\begin{tabular}{cccc}
\hline PSP-MILP & {$[\boldsymbol{M}]=1$} & {$[\boldsymbol{M}]=2$} & {$[\boldsymbol{M}]=3$} \\
\hline$k=1$ & $2.1 \mathrm{~s}$ & $2.9 \mathrm{~s}$ & $3.5 \mathrm{~s}$ \\
$k=2$ & $1247.7 \mathrm{~s}$ & $8.0 \mathrm{~s}$ & $8.2 \mathrm{~s}$ \\
NPSP-MILP & $24526.2 \mathrm{~s}$ & $16723.8 \mathrm{~s}$ & $352.3 \mathrm{~s}$ \\
\hline
\end{tabular}

Fig. 5 shows the maximum number of the used slot indexes as a function of our proposed PSPMILP jointly with Yen algorithm (Katoh et al., 1982) (with $k=1$ and $k=2$ ) in comparison with NPSP-MILP, considering 3 different simulation scenarios: (1) $|M|=3\left(z_{1}, z_{2}\right.$ and $\left.z_{3}\right) ;(2)|M|=2\left(z_{1}\right.$ and $z_{2}$ ) and (3) $|M|=1\left(z_{1}\right)$. The traffic is: (a) $v^{i j}=100 \mathrm{Gbps}$ and (b) uniformly distributed between 100, 200, 300 and $400 \mathrm{Gbps}$ for all source-destination node pairs in the small network.

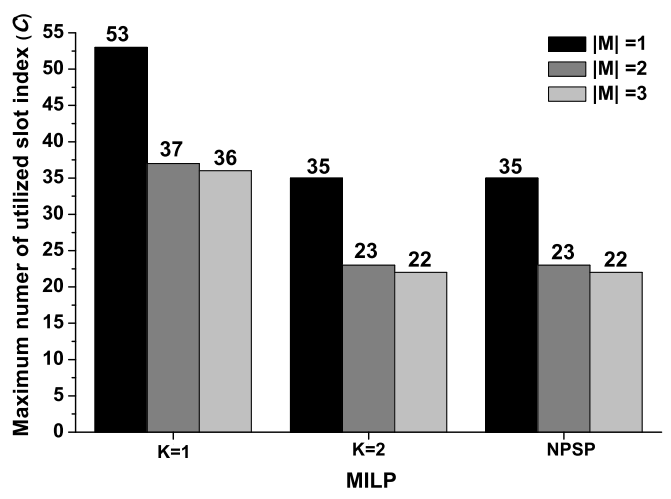

(a) $v^{i j}=100 \mathrm{Gbps}$.

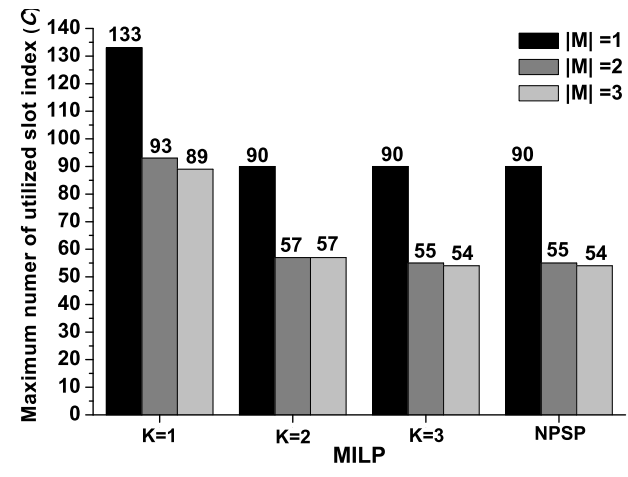

(b) Uniformly distributed between 100,200, 300 and 400 Gbps.

Fig. 5: Maximum number of the used slot indexes as a function of our proposed PSP-MILP jointly with Yen algorithm (Katoh et al., 1982) (with $k=1$ and $k=2$ ) in comparison with NPSP-MILP, considering 3 different simulation scenarios: (1) $|M|=3\left(z_{1}, z_{2}\right.$ and $\left.z_{3}\right) ;(2)|M|=2\left(z_{1}\right.$ and $\left.z_{2}\right)$ and (3) $|M|=1\left(z_{1}\right)$. The traffic is: (a) $v^{i j}=100 \mathrm{Gbps}$ and (b) uniformly distributed between 100, 200, 300 and 400 Gbps for all source-destination node pairs in a small network (as shown in Fig. 4).

It is possible to verify that the maximum number of slots is smaller for the simulation scenario that considers 3 modulation formats in both cases. Since 3 hops connect any pair of nodes in the topology, only the modulation format $z_{1}$ has been enabled for $M=1$. This modulation format can be used to establish all call requests. The modulation format $z_{2}$ also could be used. It is important to note that the PSP-MILP is not optimum, since it removes the flow conservation equation (10) and uses a limited set of pre-defined routes. Therefore, these routes may not coincide with the best routing solutions returned by the NPSP-MILP. In the results shown in Fig. 5a the PSP-MILP and NPSP-MILP performance coincide, but with the use of a traffic with different transmission bit rates, PSP-MILP required three additional slots (as shown in Fig. 5b).

Fig. 6 shows a comparison of our proposed PSP-MILP jointly with Yen algorithm (Katoh et al., 1982) (with $k=1$ and $k=2$ ), NPSP-MILP and heuristics for the small network shown in Fig. 4 with $v^{i j}=100 \mathrm{Gbps}$ and $|M|=3$. One can note that NPSP-MILP and PSP-MILP for $k=2$ have better performance sharing the first place and were able to minimize the maximum slot used. The BSR-M was in second place, BLSA-M and PSP-MILP for $k=1$ shared in third place and SPSR-M was 
the last one. These results are expected since the BLSA-M minimizes the maximum load (number of slots occupied) in every link and the BSR-M is a refinement of the BLSA-M.

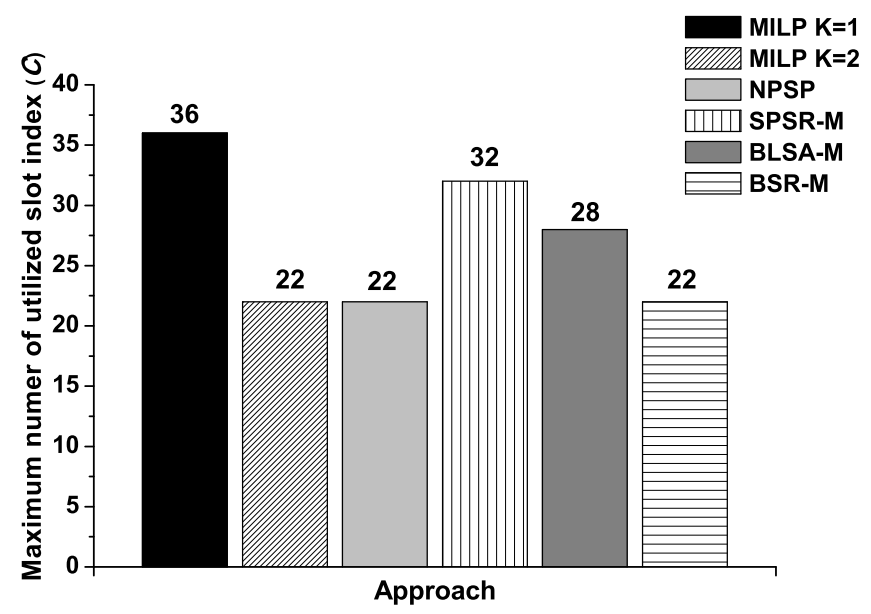

Fig. 6: Comparison of our proposed PSP-MILP jointly with Yen algorithm (Katoh et al., 1982) (with $k=1$ and $k=2$ ), NPSP-MILP and heuristics for the small network shown in Fig. 4 with $v^{i j}=100 \mathrm{Gbps}$ and $|M|=3$.

\subsection{Larger Networks}

When a large number of variables is involved, the processing time even for the PSP-MILP formulation is not viable, so, for larger networks with until 11 nodes the PSP-MILP is investigated, but apart from that only the heuristics are investigated. For larger networks simulation scenario, firstly, the simulation was performed considering our proposed PSP-MILP and the adapted heuristics (SPSR-M, BLSA-M and BSR-M) applied to well-known networks, with different number of links and nodes (RefNET2, 2020).

Table 2 presents the maximum utilized spectrum slot number for each of the analyzed large networks, considering the PSP-MILP, SPSR-M, BLSA-M and BSR-M with $|M|=4$ and $|M|=1$ modulation format.

Table 2: Maximum utilized spectrum slot number for each large network, considering our proposed PSP-MILP and the adapted heuristics (SPSR-M, BLSA-M and BSR-M) with $|M|=4$ and only $|M|=1$ modulation formats. CPU limit time ${ }^{*}=24 \mathrm{~h}, \mathrm{CPU} \operatorname{time}^{\Delta}<5 \mathrm{~min}, \mathrm{CPU} \operatorname{time}^{\dagger}<1 \mathrm{~s}$.

\begin{tabular}{|c|c|c|c|c|c|c|c|c|c|c|c|c|c|c|}
\hline \multirow[t]{2}{*}{ Network ID } & \multirow[t]{2}{*}{ Name } & \multirow[t]{2}{*}{$N$} & \multirow[t]{2}{*}{$L$} & \multirow[t]{2}{*}{$\begin{array}{c}\text { Nodal } \\
\text { Degree } \\
\text { (mean) }\end{array}$} & \multicolumn{2}{|c|}{$\begin{array}{c}\text { PSP-MILP* } \\
k=2\end{array}$} & \multicolumn{2}{|c|}{$\begin{array}{c}\text { PSP-MILP* } \\
k=1\end{array}$} & \multicolumn{2}{|c|}{ BSR-M ${ }^{\triangle}$} & \multicolumn{2}{|c|}{$\begin{array}{c}\text { BLSA-M } \\
k=2\end{array}$} & \multicolumn{2}{|c|}{ SPSR-M ${ }^{\dagger}$} \\
\hline & & & & & $|\mathrm{M}|=4$ & $|\mathrm{M}|=1$ & $|\mathrm{M}|=4$ & $|\mathrm{M}|=1$ & $|\mathrm{M}|=4$ & $|\mathrm{M}|=1$ & $|\mathrm{M}|=4$ & $|\mathrm{M}|=1$ & $|\mathrm{M}|=4$ & $|\mathrm{M}|=1$ \\
\hline 1 & VIA NET & 9 & 12 & 2.67 & 39 & 80 & 44 & 89 & 39 & 80 & 42 & 88 & 48 & 98 \\
\hline 2 & BREN & 10 & 11 & 2.20 & 54 & 116 & 54 & 107 & 54 & 107 & 60 & 116 & 68 & 132 \\
\hline 3 & LEARN & 10 & 12 & 2.40 & 58 & 107 & 54 & 107 & 54 & 107 & 58 & 113 & 70 & 141 \\
\hline 4 & ABILENE & 11 & 14 & 2.54 & 78 & 143 & 90 & 152 & 80 & 146 & 85 & 150 & 93 & 161 \\
\hline 5 & COMPUSERV & 11 & 14 & 2.55 & 46 & 98 & 62 & 125 & 47 & 100 & 60 & 122 & 71 & 133 \\
\hline
\end{tabular}

The results for $M=1$ follows the results of $M=4$. Therefore, our biggest concern will be with the results with several modulation formats available. The spectral efficiency of each modulation 
format, $\eta_{z}$, is set as $\eta_{1}=1, \eta_{2}=2, \eta_{3}=3$ and $\eta_{4}=4 \mathrm{bit} / \mathrm{s} / \mathrm{Hz}$. The maximum reach of a lightpath under each modulation format $z$ is $d_{1}=8, d_{2}=4, d_{3}=2$ and $d_{4}=1$ hop(s). For $M=4$, it is apparent from Table 2 that PSP-MILP for $k=2$ has the best performance for that set of networks, except for the network LEARN. The most interesting finding was BSR-M to have performance similar to PSP-MILP for 3 (VIA NET, BREN and LEARN) of 5 networks, but with less computational time (less than $5 \mathrm{~min}$ ). Therefore, that is a strong evidence of BSP-M could be applied for larger networks. Also there were no significant increase of number of slots when using the BLSA-M approach. However, SPSR-M did not get good results despite of CPU time to be low. Theses results, mainly regarding the simulation time, provide further support for the hypothesis of good reults for large networks with acceptable simulation time.

For networks with 12 or more nodes we used only the adapted heuristics, because our proposed PSP-MILP can take a very long time to find good solutions. Table 3 presents the maximum utilized spectrum slot number for each large network, considering SPSR-M, BLSA-M and BSR-M with $|M|=4$ and $|M|=1$ modulation formats. Usually, the SPSR-M is compared with BLSA-M. Again, load balancing was achieved by BLSA-M, but not by SPSR-M. This may be related to the fact that BLSA-M uses the longer path routing and SPSR-M the shortest path. Hence, the overall path overlapping in BLSA-M is more than SPSR-M. However, that is better to minimize the $C$.

Table 3: Maximum utilized spectrum slot number for each large network, considering SPSR-M, BLSA $-\mathrm{M}$ and BSR-M with $|M|=4$ and only $|M|=1$ modulation formats, CPU limit time* $=24 \mathrm{~h}$, CPU time ${ }^{\Delta}<5$ min, CPU time ${ }^{\dagger}<1 \mathrm{~s}$.

\begin{tabular}{|c|c|c|c|c|c|c|c|c|c|c|}
\hline \multirow[t]{2}{*}{ Network ID } & \multirow[t]{2}{*}{ Name } & \multirow[t]{2}{*}{$N$} & \multirow[t]{2}{*}{$L$} & \multirow[t]{2}{*}{$\begin{array}{c}\text { Nodal } \\
\text { Degree } \\
\text { (mean) }\end{array}$} & \multicolumn{2}{|c|}{ BSR-M ${ }^{\triangle}$} & \multicolumn{2}{|c|}{$\begin{array}{l}\text { BLSA-M }{ }^{\dagger} \\
\text { yen: } k=2\end{array}$} & \multicolumn{2}{|c|}{ SPSR-M ${ }^{\dagger}$} \\
\hline & & & & & $|\mathrm{M}|=4$ & $|\mathrm{M}|=1$ & $|M|=4$ & $|\mathrm{M}|=1$ & $|\mathrm{M}|=4$ & $|\mathrm{M}|=1$ \\
\hline 6 & vBNS & 12 & 17 & 2.83 & 94 & 170 & 106 & 186 & 120 & 221 \\
\hline 7 & CESNET & 12 & 19 & 3.17 & 69 & 140 & 78 & 160 & 80 & 169 \\
\hline 8 & ITALY & 14 & 29 & 4.14 & 52 & 112 & 63 & 132 & 101 & 198 \\
\hline 9 & ACONET & 15 & 22 & 2.93 & 98 & 192 & 106 & 207 & 121 & 224 \\
\hline 10 & GERMANY & 17 & 26 & 3.06 & 161 & 258 & 185 & 273 & 201 & 303 \\
\hline 11 & SPAIN & 17 & 28 & 3.29 & 99 & 193 & 133 & 266 & 160 & 321 \\
\hline 12 & MEMOREX & 19 & 24 & 2.53 & 293 & 427 & 381 & 536 & 419 & 564 \\
\hline 13 & CANARIE & 19 & 26 & 2.74 & 294 & 395 & 314 & 429 & 348 & 477 \\
\hline 14 & EON & 19 & 37 & 3.89 & 99 & 177 & 108 & 206 & 132 & 251 \\
\hline 15 & ARPANET & 20 & 32 & 3.20 & 186 & 298 & 211 & 332 & 211 & 350 \\
\hline 16 & PIONIER & 21 & 25 & 2.38 & 326 & 456 & 450 & 602 & 456 & 629 \\
\hline 17 & $\operatorname{cox}$ & 24 & 40 & 3.33 & 221 & 389 & 253 & 440 & 281 & 483 \\
\hline 18 & PORTUGAL & 26 & 36 & 2.77 & 479 & 589 & 737 & 911 & 823 & 985 \\
\hline 19 & RENATER & 27 & 35 & 2.59 & 396 & 550 & 582 & 866 & 686 & 1017 \\
\hline
\end{tabular}

To create a balance between SPSR-M and BLSA-M, the BSR-M works defining the problem of choosing the best combination among multiple combinations of shortest paths (MCSP) when we use the fixed routing strategy in EONs. A BSR-M fixed routing algorithm looks for a solution of the MCSP problem. For different topologies its performance was compared with both SPSR-M and BLSA-M, indicating a better result in relation to all other cases evaluated. Even for the small network under range of traffic (100 Gbps until $400 \mathrm{Gbps})$. Thus, it is possible to conclude that the BSR-M algorithm has superior performance for different kind of networks. 


\section{Conclusions}

In this paper, we proposed a novel hybrid strategy that combines MILP formulation with different alternative routing approaches that is capable to solve, simultaneously, the routing, modulation level and spectrum assignment problem in the design of elastic optical networks, considering traffic static. We considered Yen algorithm as fixed-alternative algorithm to feed PSP-MILP formulation in diverse simulation scenarios (small and intermediate sizes of networks) and three heuristics available in the literature were adapted (SPSR-M, BLSA-M and BSR-M) to compare with our proposal.

PSP-MILP formulation presents a path-link formulation and, although it cannot find the optimal solution for several cases, it finds reasonable solutions within viable simulation time. The results show the benefits of our proposal considering diverse simulation scenarios and different number of modulation formats over realistic networks in terms of simulation run time and maximum number of utilized slot indexes. We found that the path-link formulation can bring benefits in designing small and intermediate networks.

The finding that BSR-M heuristic can optimize the network design with different configuration of modulation formats add to a growing body of literature on optical networks design. The scope of study was limited in terms of physical layer, apart from modulation format constraints, it would be interesting to assess the effects of physical impairments. Additional advances can be made regarding dynamic operation of EONs, by means of flexible networking deployment. PSP-MILP can not find the optimal solution in all circumstances, but many times it can find a solution for larger networks in an acceptable time.

Acknowledgements The authors thank to CAPES and CNPq for scholarships and grants, and to UFBA, UFRB, UFPE, IFSERTAO-PE and UFCG for their educational support.

\section{Declaration}

\section{Conflict of interest}

The authors declare that they have no conflict of interest.

\section{References}

Abkenar FS, Rahbar AG (2017) Study and analysis of routing and spectrum allocation (RSA) and routing, modulation and spectrum allocation (RMSA) algorithms in elastic optical networks (EONs). Optical Switching and Networking 23:5 - 39, DOI https://doi.org/10.1016/j.osn.2016. 08.003, URL http://www.sciencedirect.com/science/article/pii/S1573427716300741

Adhikari D, Datta D, Datta R (2020) Impact of BER in fragmentation-aware routing and spectrum assignment in elastic optical networks. Computer Networks p 107167

Alyatama A, Alotaibi E, Alenezi S (2020) A continuous service model for elastic optical network. Optical and Quantum Electronics 52(7):1-19

Assis KDR, dos Santos AF, Jr RCA (2014) Optimization in spectrum-sliced optical networks. In: Optical Metro Networks and Short-Haul Systems VI, International Society for Optics and Photonics, SPIE, vol 9008, pp 102 - 109, DOI 10.1117/12.2040472, URL https://doi.org/10. $1117 / 12.2040472$

Assis KDR, Almeida RC, Waldman H, Santos AF, Alencar MS, Reed MJ, Hammad A, Simeonidou D (2019) SLA formulation for squeezed protection in elastic optical networks considering the modulation format. IEEE/OSA Journal of Optical Communications and Networking 11(5):202212 
Cavalcante MA, Pereira HA, Almeida RC (2017) SimEON: an open-source elastic optical network simulator for academic and industrial purposes. Photonic Network Communications 34(2):193201

Chatterjee B, Oki E (2020) Elastic Optical Networks: Fundamentals, Design, Control, and Management. CRC Press

Chen X, Guo J, Zhu Z, Proietti R, Castro A, Yoo SJB (2018) Deep-RMSA: A deep-reinforcementlearning routing, modulation and spectrum assignment agent for elastic optical networks. In: Optical Fiber Communications Conference and Exposition (OFC), pp 1-3

Chralyvy A (2009) Plenary paper: The coming capacity crunch. In: European Conference on Optical Communication, pp 1-1

Christodoulopoulos K, Tomkos I, Varvarigos EA (2010) Routing and spectrum allocation in OFDMbased optical networks with elastic bandwidth allocation. In: Global Telecommunications Conference (GLOBECOM), pp 1-6

Christodoulopoulos K, Tomkos I, Varvarigos EA (2011) Elastic bandwidth allocation in flexible ofdm-based optical networks. Journal of Lightwave Technology 29(9):1354-1366

Cplex II (2009) V12. 1: User's manual for cplex. International Business Machines Corporation 46(53):157

Durães GM, Soares A, Amazonas JR, Giozza W (2010) The choice of the best among the shortest routes in transparent optical networks. Computer Networks 54(14):2400 - 2409, DOI https://doi.org/10.1016/j.comnet.2010.03.010, URL http://www.sciencedirect.com/science/ article/pii/S1389128610000873

Gerstel O, Jinno M, Lord A, Yoo SJB (2012) Elastic optical networking: a new dawn for the optical layer? IEEE Communications Magazine 50(2):s12-s20

Gong L, Zhou X, Lu W, Zhu Z (2012) A two-population based evolutionary approach for optimizing routing, modulation and spectrum assignments (RMSA) in O-OFDM networks. IEEE Communications Letters 16(9):1520-1523

Gong L, Zhou X, Liu X, Zhao W, Lu W, Zhu Z (2013) Efficient resource allocation for all-optical multicasting over spectrum-sliced elastic optical networks. IEEE/OSA Journal of Optical Communications and Networking 5(8):836-847

Goścień R, Walkowiak K, Klinkowski M (2015) Tabu search algorithm for routing, modulation and spectrum allocation in elastic optical network with anycast and unicast traffic. Computer Networks 79:148 - 165, DOI https://doi.org/10.1016/j.comnet.2014.12.004, URL http://www . sciencedirect.com/science/article/pii/S1389128614004460

Hua K, Qiu Y (2020) Spectrum defragmentation based on path-switching mechanism for $1+1$ protection in elastic optical networks. In: International Conference on Optical Instruments and Technology: Optical Communication and Optical Signal Processing, International Society for Optics and Photonics, vol 11435, p 1143503

Katoh N, Ibaraki T, Mine H (1982) An efficient algorithm for k shortest simple paths. Networks 12(4):411-427

Klinkowski M, Walkowiak K (2011) Routing and spectrum assignment in spectrum sliced elastic optical path network. IEEE Communications Letters 15(8):884-886, DOI 10.1109/LCOMM.2011. 060811.110281

Klinkowski M, Walkowiak K, Jaworski M (2011) Off-line algorithms for routing, modulation level, and spectrum assignment in elastic optical networks. In: International Conference on Transparent Optical Networks, pp 1-6

Lechowicz P, Walkowiak K (2016) Genetic algorithm for routing and spectrum allocation in elastic optical networks. In: European Network Intelligence Conference (ENIC), pp 273-280

Lira CN, Júnior RA, Chaves D, Waldman H, Assis K (2020) Meta-heuristic procedure for enhanced spectrum fragmentation assessment in elastic optical networks. Journal of Communication and Information Systems 35(1):333-337 
López V, Velasco L, et al. (2016) Elastic optical networks. Architectures, Technologies, and Control, Switzerland: Springer Int Publishing

Mavridopoulos SB, Beletsioti G, Nicopolitidis P, Papadimitriou GI, Varvarigos E (2020) Hop distance-based bandwidth allocation technique for elastic optical networks. International Journal of Communication Systems 33(8):e4360, DOI 10.1002/dac.4360, URL https://onlinelibrary . wiley.com/doi/abs/10.1002/dac. 4360

Mesquita LAJ, Assis KDR, Almeida RC (2020) Multi-period traffic on elastic optical networks planning: alleviating the capacity crunch. The Journal of Supercomputing pp 1-24

Oner P (2016) Routing and spectrum allocation in static fiber optic networks. In: Master of Science - Graduate School of Engineering and Science of Bilkent University

Ramaswami R, Sivarajan K, Sasaki G (2009) Optical networks: a practical perspective. Morgan Kaufmann

RefNET2 (2020) Real-world reference networks. URL http://www.av.it.pt/anp/on/refnet2. html

Ríos I, Bogado CM, Pinto-Roa DP, Barán B (2018) A serial optimization and link-oriented based routing approach for RMLSA in elastic optical networks: A comparison among ILP based solution approaches. In: Proceedings of Latin America Networking Conference, Association for Computing Machinery, New York, NY, USA, LANC '18, pp 48-55, DOI 10.1145/3277103.3277125, URL https://doi.org/10.1145/3277103.3277125

Savory SJ (2014) Congestion aware routing in nonlinear elastic optical networks. IEEE Photonics Technology Letters 26(10):1057-1060

Varvarigos EA (2013) An introduction to routing and wavelength assignment algorithms for fixed and flexgrid. In: Optical Fiber Communication Conference, Optical Society of America, pp OW1H-4

Velasco L, Klinkowski M, Ruiz M, Comellas J (2012) Modeling the routing and spectrum allocation problem for flexgrid optical networks. Photonic Network Communications 24(3):177-186

Waldman H (2018) The impending optical network capacity crunch. In: International Optics and Photonics Conference (SBFoton IOPC), pp 1-4

Wang X, Brandt-Pearce M, Subramaniam S (2015) Impact of wavelength and modulation conversion on translucent elastic optical networks using MILP. IEEE/OSA Journal of Optical Communications and Networking 7(7):644-655

Wang Y (2019) A research on spectrum defragmentation algorithms in elastic optical network. In: World Symposium on Communication Engineering (WSCE), IEEE, pp 78-81

Wang Y, Cao X, Pan Y (2011) A study of the routing and spectrum allocation in spectrum-sliced elastic optical path networks. In: Proceedings IEEE INFOCOM, pp 1503-1511

Xuan H, Wang Y, Xu Z, Hao S, Wang X (2017) New bi-level programming model for routing and spectrum assignment in elastic optical network. Optical and Quantum Electronics 49(5):186

Yaghubi-Namaad M, Rahbar AG, Alizadeh B (2018) Adaptive modulation and flexible resource allocation in space-division-multiplexed elastic optical networks. Journal of Optical Communications and Networking 10(3):240-251

Yin Y, Zhang H, Zhang M, Xia M, Zhu Z, Dahlfort S, Yoo SJB (2013a) Spectral and spatial 2D fragmentation-aware routing and spectrum assignment algorithms in elastic optical networks [invited]. IEEE/OSA Journal of Optical Communications and Networking 5(10):A100-A106, DOI 10.1364/JOCN.5.00A100

Yin Y, Zhang M, Zhu Z, Yoo SJB (2013b) Fragmentation-aware routing, modulation and spectrum assignment algorithms in elastic optical networks. In: Optical Fiber Communication Conference and Exposition and the National Fiber Optic Engineers Conference (OFC/NFOEC), pp 1-3

Zang H, Jue JP, Mukherjee B, et al. (2000) A review of routing and wavelength assignment approaches for wavelength-routed optical wdm networks. Optical networks magazine 1(1):47-60

Zhao J, Wymeersch H, Agrell E (2015) Nonlinear impairment-aware static resource allocation in elastic optical networks. Journal of Lightwave Technology 33(22):4554-4564 